\title{
Dossiê: "Racismos"
}

\section{LA MALATTIA CHIAMATA EUROPA. CONTROVERSIA SULL'ISLAMIZZAZIONE IN EUROPA E RINASCITA DEL NAZIONALISMO}

\author{
The Sickness Called Europe. Islamization of Europe in Dispute \\ and the Rebirth of Nationalism
}

Enzo Pace ${ }^{*}$

\begin{abstract}
Riassunto. L'articolo si focalizza sui nuovi movimenti nazionalisti che si stanno affermando politicamente nell'Unione Europea e sulla battaglia che essi conducono contro la diversità religiosa, sempre più visibile nelle società europee, ritenuta una minaccia all'identità cristiana dell'Europa. L'islam è, perciò, diventato, un oggetto polemico (nel senso del polemos, la guerra ideologica che divide drammaticamente la società) nel discorso politico. Tramite tale discorso si tende a costruire una narrazione collettiva convincente: una società troppo diversa dal punto di vista religioso non è sostenibile.
\end{abstract}

Parole chiave: Unione Europea; diversità religiosa; Islam; controversia sull'islamizzazione; nazionalismi.

\begin{abstract}
The article focuses on the new nationalist movements that are emerging in the European Union and on ideological the battle they are leading against religious diversity, increasingly visible in European societies. They consider that a threat to the Christian identity of Europe. Islam has therefore become a polemical object (in the sense of polemos, the ideological battle that divides society drastically) in political discourse. Through this discourse, neo-nationalist tend to construct a convincing collective narrative: a society that is too different from the religious point of view is not sustainable.
\end{abstract}

Keywords: European Union; Religious Diversity; Islam; Islamization in dispute; Nationalism.

\footnotetext{
*Professore di sociologia della religione nell'Università di Padova - Italia. Padova, Italia.
} 


\section{Introduzione}

Preferisco la mia famiglia agli amici, gli amici ai miei vicini, i miei compatrioti agli Europei. Così parlò Jean-Marie Le Pen, ex-leader del Front National in Francia il 9 dicembre 2006. A seguire, sua figlia, Marine, che è diventata presidente dello stesso partito, nel 2011, non senza un sorprendente strappo ideologico da suo padre, ha più volte ripetuto che: $i$ grandi principi del Fronte nazionale sono sempre gli stessi: ristabilire l'autorità dello Stato e della sovranità nazionale; inoltre, assicurare la perennità della nostra civiltà.

Se confrontiamo queste citazioni con altre, riferite a gente comune, a delle donne europee, cittadine belghe, danesi, francesi, inglesi e italiane, che hanno accolto l'invito da parte del New York Times (Dremaux, 2016) di esprimere il loro parere sull'affaire burkini, si comprende la distanza fra l'idea di Europa che hanno queste ragazze intervistate rispetto a quella della famiglia Le Pen.

Quando la questione del burkini si è posta, io ero felice per mia sorella, che era in vacanza e che avrebbe potuto finalmente giocare con $\mathrm{i}$ suoi bambini sulla spiaggia invece di stare seduta all'ombra. Quando è esploso il caso, ho detto a mia sorella 'non ti preoccupare; è solo uno sparuto gruppetto di persone che non hanno niente da fare che passare il tempo a odiare qualcuno...', ma poi mi sono ricreduta quando ho costatato che I'Europa era contro. Com'è possibile che nel mondo moderno, prendere il sole nudi sia accettato e coprirsi con i propri abiti non lo sia? (Dina Srouji, 23 anni, Lebekke, Belgio)

Tutto ciò mi ricorda il mio primo giorno di scuola subito dopo la legge che bandiva lo hijab a scuola. La mia insegnante mi obbligò a togliermelo di fronte a tutta la classe. lo mi sentii umiliata. Oggi il mio cuore è ancora una volta spezzato. Ho visto alla TV questa donna obbligata togliersi il burkini in spiaggia e mi sono chiesta: quando finirà tutto ciò? (Hajer Zennou, 27 anni, Lione, Francia)

Ho paura che un giorno dovrò indossare la mezzaluna gialla sui miei abiti come la stella di David per gli ebrei molti anni fa. (Charlotte, Monnier, 23 anni, Toulouse, Francia)

È curioso vedere che sia proibito il burkini mentre i cani possono nuotare in acqua. Sono sorpresa perché $i$ cani hanno più diritti di una donna velata. (Samia Fekih, 36 anni, Parigi, Francia)

Spesso dopo che ho detto a qualcuno che sono siriana, questi mi chiede se sono anche musulmana? Ma voi chiedereste a una persona dopo dieci minuti con cui parlate qual è la sua fede? Mi chiedo, siamo davvero uomini e donne libere? Non lo so più. (Aya Homsi, 32 anni, Bologna, Italia)

II plesso di brevi frasi appena riportate riassume bene i contorni di un conflitto culturale che negli ultimi quindici anni è diventato sempre più aspro. All'aumentata diseguaglianza economica, provocata dalla crisi finanziaria del 2008, si è aggiunto il crescente allarme sociale per l'insicurezza nella vita 
quotidiana, causata dai gruppi armati d'ispirazione musulmana che usano il metodo terroristico per raggiungere i loro obiettivi militari e politici. Due elementi questi ultimi di crisi che sono stati ulteriormente esasperati dalle migrazioni da zone del mondo afflitte da guerre, disastri ambientali, crescente miseria ed esistenza di dittature. Il tutto sullo sfondo storico dell'eclissi delle principali, grandi ideologie politiche dell'Otto-Novecento. Disincantati e incattiviti ${ }^{1}$, cresce il numero di cittadini che non ha più fiducia nelle élite politiche accusate di essere solo i curatori degli interessi di ristrette clientele economiche e finanziarie. II termometro politico registra, da un'elezione all'altra in Europa, I'allargarsi del consenso verso partiti politici che risvegliano, via democratica, l'unica ideologia che sembra sopravvissuta alla crisi delle grandi ideologie: un nazionalismo etnico e religioso che nel cuore stesso dell'Europa è disposto a sacrificare la cultura dei diritti, di tradizione liberale, e lo spirito di solidarietà sociale, d'ispirazione cristiana e socialista, in difesa dell'identità nazionale. Tutto ciò è stato ben sintetizzato da Papa Francesco nel novembre 2014 al Parlamento di Strasburgo:

Questa famiglia di popoli, lodevolmente diventata nel frattempo più ampia, in tempi recenti sembra sentire meno proprie le mura della casa comune, talvolta innalzate scostandosi dall'illuminato progetto architettato dai Padri. Quell'atmosfera di novità, quell'ardente desiderio di costruire I'unità paiono sempre più spenti; noi figli di quel sogno siamo tentati di cedere ai nostri egoismi, guardando al proprio utile e pensando di costruire recinti particolari. Tuttavia, sono convinto che la rassegnazione e la stanchezza non appartengono all'anima dell'Europa e che anche le difficoltà possono diventare promotrici potenti di unità. (Papa Francesco, 2014)

Di fronte al mondo in casa, in molte società dell'Unione Europea cresce il sentimento di ostilità nei confronti di un cambiamento che diventa sempre più visibile. Esso si mostra, da un lato, nella transizione demografica verso società sempre più stratificate e differenziate per lingue, culture, religioni, stili e modelli di vita individuali e collettivi e, dall'altro, nella conseguente riconfigurazione dei luoghi pubblici, divenuti spazi di coabitazione fra cittadini portatori di tante e diverse diversità, spesso inedite e inattese. Tutto ciò suscita insicurezza in tanti concittadini che tendono a pensare di non essere più a casa propria, di essere diventati senza dimora, costretti a convivere con altrettanti soggetti senza fissa dimora, provenienti da mondi lontani. Su questi timori diffusi, tuttavia, stanno accumulando un capitale di consenso nuovi partiti, prevalentemente nazionalisti, che offrono come risposta rassicurante una rappresentazione della

\footnotetext{
Chi ha parlato di cittadino incattivito è stato Ulrich Beck. La società del rischio. Roma: Carocci, 2000. Sulle cause che hanno prodotto una crescente diseguaglianza economica, che a sua volta, ha reso incerto il destino sociale di ciò che un tempo erano chiamati i ceti medi, cfr. anche Thomas Piketty. Il capitale del XXI secolo. Milano: Bompiani, 2014.
} 
realtà che tende o a semplificarne la complessità oppure a polarizzarla in modo tale che sia facile trovare il capro espiatorio di tutti i mali che affliggono una società. Un vecchio e ben collaudato meccanismo psico-sociale, ben studiato da quanti hanno riflettuto sul sorgere della società di massa nel Novecento ${ }^{2}$.

Il nuovo nazionalismo europeo, che è chiamato anche da alcuni populista ${ }^{3}$, non è certo tutto omogeneo; varia in relazione al respiro lungo della storia di ciascun popolo: una cosa è il risorgente nazionalismo ungherese, polacco o slovacco, che si forma in Paesi che hanno cercato di superare il modello di Stato di tipo sovietico, un'altra cosa è l'insorgenza di movimenti e partiti d'ispirazione nazionalista in Francia o in Danimarca e così via. II nazionalismo contemporaneo non è un abito fatto su misura unica per tutte le società, dove i partiti di cui stiamo parlando sono già al potere o aspirano ad andarci. Inoltre, ci sono altri movimenti e gruppi che non sono riconducibili immediatamente all'ideal-tipo del nazionalismo, che piuttosto sembrano riprendere alcuni motivi dell'ideologia politica di tipo giacobino ${ }^{4}$, secondo cui il popolo è buono, mentre le élites sono corrotte, da cui consegue che la rappresentanza va perciò soppressa e superata da rapporti diretti, trasparenti, con il popolo.

Ciò che mi propongo di fare nelle pagine che seguono, è di focalizzare l'attenzione del lettore sul significato del polemos attorno alla religione oggi in Europa e, in particolare, su una religione che è rappresentata, soprattutto nei movimenti nazionalisti, come la vera minaccia all'identità degli europei. L'intento è di mostrare come l'islam sia divenuto, grazie anche alla strategia del terrore organizzata dai gruppi di lotta armata che dicono di ispirarsi al vero islam, un oggetto del discorso pubblico che tende a rappresentare l'Europa come una società diventata troppo diversa e che tale diversità metta in discussione i fondamenti culturali, religiosi e morali della stessa civiltà europea.

\footnotetext{
2 Alludo, in particolare, agli studi ormai classici, rispettivamente, di Elias Canetti. Massa e potere. Milano: Adelphi, 1981; José Ortega Y Gasset. La ribellione delle masse. Bologna: Il Mulino, 1974; Gustave Le Bon. Psicologia delle folle. Milano: Tea, 2004; George L. Mosse. La nazionalizzazione delle masse. Bologna: II Mulino, 1975; David Riesman. La folla solitaria. Bologna: Il Mulino, 1976. Sull'attualità del pensiero di Ortega y Gasset cfr. Monica Simeoni. Una democrazia morbosa. Roma: Carocci, 2013 e della stessa autrice (a cura di). Europa/Europe. Roma: Carocci, 2015.

3 Si vedano, ad esempio, fra gli altri, Guy Hermet. Le populisme dans le monde. Paris: Fayard, 2001; Piero Ignazi. Forza senza legittimità. Roma-Bari: Laterza, 2012; Ernesto Laclau. La ragione populista. Roma-Bari: Laterza, 2008; Alfio Mastropaolo. La democrazia è una causa persa?. Torino: Bollati Boringhieri, 2011; Cas Mudde, Cristóbal Kaltwasser. Populism in Europe and the Americas. New York: Cambridge University Press, 2011; Giuliano Santoro. Un Grillo qualunque. Roma: Lit Edizioni, 2012; Pierre-André Taguieff. L'illusione populista. Milano: Bruno Mondadori, 2003 e Idem. Le nouveau National-populisme. Paris: CNRS Éditions, 2012; Marco Tarchi. L'talia populista. Bologna: Il Mulino, 2003.

4 Sul giacobinismo storico e contemporaneo cfr. François Furet. Jacobinism. In: François Furet, Mona Ozouf (eds.). Dictionnaire critique de la Révolution française. Paris: Flammarion, 1988; Massimo Luigi Salvadori, Nicola Tranfaglia (a cura di). Il modello politico giacobino e le rivoluzioni. Firenze: La Nuova Italia, 1984; Shmuel Eisenstadt. Fondamentalismo e modernità. Eterodossie, utopismo, giacobinismo nella costruzione dei movimenti fondamentalisti. Roma-Bari: Laterza, 1994.
} 


\section{Il risorgente nazionalismo}

Il processo di unificazione politica dell'Europa si è fatto incerto da almeno un decennio. I tanti mali che affliggono I'Unione Europea, nell'ora presente, riguardano non solo l'economia, la tenuta della democrazia liberale, I'evaporazione dei partiti politici di lunga memoria storica e il rischio sempre più reale di perdere un'intera generazione a causa della persistente crisi economicofinanziaria iniziata nel 2008. A tutto ciò si aggiunge un sentimento diffuso che la crisi certamente contribuisce ad alimentare. Sto facendo riferimento alla percentuale crescente di cittadini europei che è convinta che nella casa comune non sia più possibile vivere assieme a chi è ritenuto troppo diverso da noi. La casa comune appare come una gabbia, dove forzatamente saremmo costretti a stare con altri, percepiti come talmente differenti da noi da farli apparire del tutto incompatibili. In vari partiti politici anti-europeisti, infatti, il riferimento alle radici cristiane o alla tradizione religiosa di maggioranza - dal partito dei Veri Finlandesi alla nuova Lega Nord italiana, sempre più filo-lepenista - serve a marcare la distanza ritenuta incolmabile con I'islam. Parlano di religione, ma in realtà tali partiti esprimono a voce alta una domanda di senso collettivo, che una parte non più irrilevante dei cittadini dell'Unione si pone: chi siamo e com'è possibile una solidarietà sociale fra estranei per riprendere un efficace titolo di un testo di Jürgen Habermas (1997)? È la stessa domanda, del resto, che, interpretando il disagio intellettuale di una parte dell'ambiente conservatore americano, si era posto nell'ultimo suo saggio, Samuel Huntington (2004) II problema, in tal caso, secondo lo scienziato politico americano, di compatibilità con l'ideologia wasp non sarebbe rappresentato dall'islam, ma dal cristianesimo di terza generazione, quello, per la precisione, di tendenza neo-evangelica e neo-pentecostale dei latinos e degli asiatici (Pace, Butticci, 2010; Lucà Trombetta, 2012).

Si parla di religione, dunque, per parlare di noi stessi. Sennonché noi europei pensavamo almeno di non dover fare più i conti con i conflitti di tipo religioso. Dopo la Shoa soprattutto, per restare a ieri, e dopo le lunghe guerre di religione, per riandare molto indietro nel passato. Sembrava con la caduta de Muro di Berlino che l'utopia della nuova casa comune europea dall'Atlantico agli Urali fosse a portata di mano. Le religioni secolari del XIX secolo (dal comunismo ai vari fascismi) apparivano al tramonto e gli Stati etici definitivamente usciti di scena.

Le due date-cerniere, la caduta del Muro di Berlino (1989), da un lato, e l'attentato alle Torri Gemelle di New York (2001), dall'altro, con il corollario della sequenza degli attacchi terroristici, verificatisi rispettivamente a Madrid nel 2004, a Londra nel 2005 e poi in Francia fra il 2012 e il 2015 delimitano una sorta di secolo ultra-breve. Eric Hobsbawm (1994), come si ricorderà, aveva fissato i limiti del Ventesimo secolo tra il 1914 (I'attentato all'imperatore Francesco 
Ferdinando a Sarajevo) e il 1992 (il discorso di François Mitterand nella stessa città per invocare una pace duratura nei Balcani). Il terzo millennio si colloca a cavallo fra due secoli e sembra anch'esso doversi consumare rapidamente fra la distruzione di un muro e la costruzione di tanti nuovi muri in Europa.

Lo spirito dell'Ottantanove, sorto dalle macerie del Muro berlinese, finisce presto di soffiare. I Balcani sono ancora una volta un laboratorio a cielo aperto, dove vecchie idee politiche tornano a vivere, anticipando tendenze che poi accadranno altrove. Da quel laboratorio, infatti, durante l'ultimo ciclo delle guerre del 1991-1999 (Pirjevec, 2001) è resuscitata l'idea della necessità per i popoli di avere una terra, una lingua, una fede (religiosa), un'unica nazione che faccia sintesi di tutto ciò. La diversità di lingua, di religione e di origine è apparsa nelle terre degli Slavi del sud improvvisamente insostenibile. La pulizia etnica è diventata la pratica sociale che furiosamente misconosceva l'amico di ieri e armava, l'uno contro l'altro, popoli che erano riusciti a convivere per un relativo lungo tratto di tempo.

Nel secolo brevissimo le politiche d'identità hanno contribuito a far risorgere gli etno-nazionalismi così come i nazionalismi religiosi (Pace, 2004) ${ }^{5}$. Spesso i due movimenti si sono fusi, soprattutto quando nell'immaginario collettivo di molti europei è apparso un nuovo nemico - l'islam - che ha ben presto rimpiazzato il fantasma del comunismo, sepolto ormai sotto le macerie del Muro di Berlino. La politica d'identità è il nome che diamo a un movimento sociale e politico, che si sforza di affermare l'esistenza di valori comuni e di pretenderne la piena trasfigurazione negli ordinamenti che governano la società stessa, anche quando in essa abitano minoranze culturali e religiose diverse dalla maggioranza. L'identità, in questo modo, è definita da parte di chi si fa portavoce della maggioranza come un dato naturale che fonda e legittima la struttura di governo della società; perciò, essa è vista come la fonte ultima della legittimità di uno Stato: una norma assoluta, non riducibile, che viene prima delle norme, dunque, sacra, non negoziabile. Poiché l'identità è immaginata come un dato-per-scontato, un muro portante delle pareti domestiche del mondo della vita degli individui, le religioni costituiscono il supplemento di senso di cui le politiche d'identità hanno bisogno per rafforzare l'azione sociale. Così facendo le religioni rafforzano l'idea che esista un mito di fondazione delle identità collettive, trasformandosi, spesso, in interpreti fedeli e ancelle premurose della solidarietà organica di un'etnia. Esse, in altre parole, lasciano immaginare che esista un'unità profonda in un popolo o in gruppo umano che vada esaltata e difesa, anche in nome di un dio o di un valore sacro. Laddove il rinforzo reciproco fra religione e politica d'identità si realizza, il conflitto è la

\footnotetext{
5 Sul panorama multireligioso in Europa cfr. anche Frédéric Lénoir. Le bouddhisme en France. Paris: Fayard, 1999; Lionel Obadia. Il buddismo in Occidente. Bologna: Il Mulino, 2009.
} 
linfa vitale che ossigena il cervello sociale dei movimenti collettivi e dei gruppi a base fideistica. Le religioni, divenendo in tal modo ideologie etniche, finiscono per negare la loro pretesa d'essere depositarie di valori universali (come la pace, ad esempio); esse si particolarizzano, invece, finendo, appunto, per prendere parte diretta alla contesa che oppone un'identità etnica a un'altra. Il laboratorio balcanico ha anticipato i temi che oggi occupano una parte dell'agenda politica europea e che suscitano conflitti e tensioni in quasi tutte le società europee. Almeno in tutte quelle che, nel ciclo del movimento migratorio, iniziato in Europa (è sempre bene rammentarlo) proprio subito dopo la costruzione del Muro di Berlino (nel 1961), hanno visto mutare gradualmente, ma profondamente, la geografia culturale e religiosa delle loro rispettive società. Invece di parlare di società multiculturale - poiché tale aggettivo è oggi fonte di controversie ideologiche -, preferiamo descriverle, dunque, come società caratterizzate da inedite diversità culturali e religiose (Pace, 2013).

\section{La super-diversità religiosa in Europa}

Un viaggiatore distratto e frettoloso che, ad esempio, partendo dal cuore della Valle Padana in Italia si spingesse nelle terre di mezzo in Gran Bretagna, risalendo, indifferentemente, o la Germania o i Paesi Bassi e poi discendesse lungo le vie che lo portano sino a Granada e per le Terre della Mora incantata, fra Spagna e Portogallo, non sarebbe certo colpito da edifici di culto diversi da quelli a lui più familiari, le chiese con i campanili. Non si accorgerebbe, a occhio nudo (senza gli occhiali con cui guardiamo un ambiente a noi familiare, appunto, come può essere una città o un villaggio dove c'è una chiesa grande o piccola che sia, poco importa) di come il territorio religioso europeo si stia popolando di tanti nuovi templi. Gli uffici turistici dovranno presto aggiornare il viaggiatore con una nuova mappa socio-religiosa dell'Europa. Solo arrivando in Gran Bretagna egli realizzerebbe finalmente, a occhio nudo, che accanto ad una chiesa (anglicana o cattolica o protestante) sono sorti templi hindu, gurudwara (templi sikh) e poi sono state costruite moschee (secondo i canoni classici dell'architettura e dell'arte d'ispirazione musulmana) e, ancora, si sono moltiplicati centri di meditazione che fanno riferimento a maestri buddisti delle varie scuole di pensiero che questa filosofia e sapienza spirituale ha conosciuto nel corso del tempo (Allievi, 2010; Frisina, 2007).

Allo stesso modo, I'inedito pluralismo religioso europeo che comincia a prendere forma in diverse società non va tuttavia sovradimensionato, per una serie di ragioni che è bene tenere presenti. Se per un verso le proiezioni demografiche indicano per il 2050 una crescita continua di comunità di persone con diversi orientamenti religiosi, per un altro, la loro consistenza reale è ancora relativamente limitata. Una delle più estese, quella musulmana ad esempio, non supera mediamente il 3\% delle popolazioni dei principali 
Paesi europei che hanno conosciuto l'arrivo d'immigrati sin dagli inizi degli anni Sessanta del secolo appena trascorso. Non a caso il solo Paese europeo, di recente aggregato all'Unione, che presenta una percentuale elevata di musulmani (12\%) è la Bulgaria. Inoltre, giacché nelle principali nazioni europee con milioni di cittadini di fede musulmana, siamo di fronte in realtà a seconde e terze generazioni, come accade rispettivamente in Belgio, Olanda, Francia, Gran Bretagna e Germania (mentre, Italia, Spagna e Portogallo sono ancora ferme, ma ancora per poco, alle seconde), è ben difficile stabilire con esattezza quante di queste persone abbiano mantenuto forte il legame di appartenenza con le loro rispettive religioni di nascita e in quante diverse forme l'identità e l'esperienza religiosa si esprima, quando entrambe siano conservate nel passaggio da una generazione all'altra.

Insomma ci si continua a rappresentare l'Europa o come prevalentemente cattolica o protestante, ma in realtà lento pede, la tartaruga del cambiamento religioso avanza, rendendo tale rappresentazione sociale sempre meno realistica ${ }^{6}$. Non lo è più da qualche tempo, del resto, se soprattutto si pone attenzione ai risultati delle ricerche longitudinali dell'European Values Studies: esse hanno messo in luce che le storiche divisioni fra cattolici e protestanti siano state di gran lunga colmate non tanto dal dialogo ecumenico, ma dai processi di secolarizzazione che hanno avvicinato nei comportamenti socioreligiosi persone che appartengono a una differente religione di nascita. Le ricerche condotte in Svizzera, inoltre, dapprima da Campiche (1992) e, più recentemente, da Stolz (2016), dimostrano come le frontiere religiose siano state gradualmente abbattute se si guardano gli stili di vita e si ricostruiscono le mappe cognitive delle persone sondate.

Da qui nasce il rompicapo del riconoscimento della diversità religiosa. Le società europee, in modo vario, appaiono in alcuni casi sull'orlo di una crisi di nervi. La Cancelliera tedesca, Angela Merkel, il 16 ottobre del 2010, in un intervento a un convegno dei giovani della $\mathrm{CDU}$, ha dichiarato, facendo eco a quanto aveva detto qualche giorno prima l'ex-leader conservatore Cameron: "Il modello multiculturale è totalmente fallito, la Germania non ha manodopera qualificata e non può fare a meno degli immigrati, ma questi si devono integrare e devono adottare la cultura e i valori tedeschi". Un de profundis del multiculturalismo? Non è la sola personalità politica a sostenere che le politiche d'integrazione dei nuovi cittadini di origine straniera, fondate sul riconoscimento delle loro plurime diversità culturali e religiose, sembrano aver fallito gli obiettivi che esse si promettevano di perseguire, laddove sono state assunte e perseguite con coerenza. Il riferimento è al modello elaborato e messo in pratica in Gran Bretagna o nei Paesi Bassi.

${ }_{6}$ Cfr. per questa metafora Enzo Pace. Achilles and the tortoise. Social Compass, v. 6, p. 315-331, 2013. 
La via tedesca che mirava a scoraggiare il desiderio d'integrazione di tanti immigrati, soprattutto provenienti dall'Anatolia, rappresentava almeno sino al 1990 un contro-modello. La sua specificità era caratterizzata, da un lato, dalla figura giuridica del lavoratore ospite (gastarbeiter) e dall'altro, dal principio costituzionale dello jus sanguinis che regolava l'accesso alla cittadinanza. Dopo il crollo del Muro, anche la Germania ha iniziato un profondo processo di revisione, prendendo atto della realtà di una popolazione di origine straniera che non poteva essere mantenuta in una sorta di limbo giuridico e costituzionale. La convinzione di molti politici che hanno guidato i principali partiti della Germania fra il 1990 e il 2001 era di segno diverso rispetto al passato: riformare la legge sulla cittadinanza e favorire una nuova forma di coesione sociale, che mirasse a favorire la diversità nell'uguaglianza e nel rispetto dei diritti fondamentali della persona. II 2001 costituisce una data cerniera per tutti, ma ancor più per la società tedesca, nel senso che l'adozione di una politica di coesione sociale che tende a includere le differenze, sembra aver subito una battuta di arresto.

\section{Conclusione: una solidarietà fra estranei?}

Come altri leader del resto in Europa, anche la Merkel sente montare nell'opinione pubblica un sentimento di ostilità verso lo straniero, anche quando ormai quest'ultimo, straniero non lo è più, pensando al fatto che ormai ci sono almeno due generazioni di mezzo fra i primi immigrati e i loro discendenti (in particolare, come nel caso dei turchi). Nelle retoriche dei leader politici, in realtà, si celano questioni molto più complesse che riguardano diversi aspetti della vita sociale. Essi possono essere racchiusi nella domanda assillante cui la politica in Europa non è stata sinora in grado di rispondere in modo adeguato: come sia possibile lo sviluppo di un modello interculturale sostenibile, che, per un verso, non dissipi le identità culturali e lo spirito dello Stato di diritto, di lungo respiro nella storia europea, e, per un altro, favorisca l'uguaglianza (o le pari opportunità) delle persone le quali hanno inscritto nella loro biografia una pluralità d'identità.

Le preoccupazioni di molti leader in Europa, inoltre, nascono anche in considerazione della crescita di movimenti e gruppi politici che programmaticamente sono contro una società che, ai loro occhi, si sta estraniando, perdendo le proprie radici culturali e religiose a causa di un'invasione di stranieri, che mostrano di non volersi assimilare ai costumi e agli ordinamenti giuridici vigenti. Sovente la figura che maggiormente riassume le due dimensioni dell'estraneo in casa nostra e dell'estraneo che finirà per imporci la sua religione e le sue leggi è quella del musulmano.

$\mathrm{E}^{\prime}$ sufficiente guardare, d'altro canto, i risultati dell'ultimo rapporto dell'European Monitoring Centre on Racism and Xenophobia del 2014. Esso mostra come sia cresciuto fra la popolazione dell'UE il sentimento di ostilità nei 
confronti delle diversità culturali che hanno poi preso corpo (sociale) negli ultimi venti anni in Europa. Un quarto, infatti, dei cittadini europei non condivideva I'affermazione "la diversità etnica, culturale, religiosa è un elemento positivo che arricchisce una società" e i due terzi (con un incremento del 50\% rispetto al 1997) è convinto che "la società multiculturale ha ormai raggiunto il suo limite", oltre il quale essa diventa insostenibile".

L'interrogativo che sorge, quando si osservano questi dati, è se esista una correlazione plausibile fra l'aumento della diversity-rate (il tasso di diversificazione o di pluralità inedita che stiamo conoscendo), riconducibile al flusso degli immigrati nei diversi cicli che abbiamo conosciuto in Europa, e, I'hostility-rate (o l'indice di sfiducia crescente sino all'ostilità aperta) nei confronti delle diversità.

Da un'indagine condotta da un gruppo di ricercatori dell'Università di Nijmegen (Lubbers, Gijsberts, Schepeers, 2002) su venti Paesi dell'OCSE, si ricava che: a) il tasso di xenofobia ha una relazione statisticamente significativa con il tasso di disoccupazione ma b) il primo ha più probabilità di crescere quando in una società è presente un partito o un movimento politico che fa risalire i disagi economici e i malesseri sociali non solo alle politiche (fallimentari ai loro occhi) dei governi nazionali, ma anche alla presenza crescente di stranieri, per definizione, non integrabili, anzi incompatibili con i valori originari di un popolo (cultura, religione, lingua, usi e costumi). Infatti, se nei Paesi, dove non sono presenti tali partiti, il tasso di xenofobia nell'opinione pubblica arriva al $5 \%$, nell'altra metà supera il 30\%.

Se, infine, allineiamo i risultati conseguiti dai partiti anti-europeisti, ci si rende conto sia dell'ampiezza del fenomeno sia dell'allarme crescente fra i leader politici europei. Il popolo - una moltitudine crescente di cittadini incattiviti non ne può più di discorsi sulla società multiculturale e rivendica il diritto, dunque, di decidere direttamente se e fino a che punto la diversità può essere riconosciuta: è così che si sintetizza il discorso del nuovo populismo europeo. $\mathrm{E}^{\prime}$ così che l'apertura di una moschea o la costruzione di un minareto diventa oggetto del polemos politico e s'invoca il diritto da parte delle popolazioni autoctone di decidere per il sì o per il no. Il referendum sui minareti in Svizzera del 2009 l'ha mostrato in modo palese.

Le società europee sono chiamate a misurarsi, dunque, con un ambiente sociale molto più differenziato da come l'era sino a cinquanta anni fa. La migrazione di popolamento ha lentamente mutato la composizione sociodemografica delle nostre società, ha alterato il rapporto fra cittadinanza e nazionalità e ha reso più articolato, dal punto di vista linguistico, culturale e 
religioso, il nostro lessico familiare con cui classificavamo la realtà che ci circonda. Crescono sempre più, infatti, in esse i tanti cittadini che hanno un'identità col trattino (anglo-pakistano-musulmano; italo-sikh; franco-algerino; marocchinobelga; turco-tedesco ecc.), un'identità plurima che non può essere altrettanto naturalmente ridotta a una sola. Cittadini col trattino senza fissa dimora per quanto riguarda le loro origini multiple. Le nuove generazioni sono già oggi frutto di matrimoni misti, per cui esse possono aggiungere altri trattini al loro profilo socio-culturale: anglo-pakistano-anglicano-musulmano; italo-sikhcattolico; turca-tedesca-musulmana-protestante e così via. Abiti del cuore più larghi di quelli che indossavano interiormente le generazioni del baby-boom europeo. Un'inedita complessità che trova I'Unione Europea relativamente impreparata a reggere le conseguenze che la diversità culturale e religiosa ha sull'equilibrio dei sistemi sociali.

Una trasformazione sociale e culturale di questa portata non è facile da metabolizzare. I cambiamenti sociali richiedono tempi lunghi per essere capiti e accettati. In particolare, quando tali cambiamenti toccano gli abiti del cuore di persone abituate a pensare alla realtà in cui esse vivono come le pareti domestiche della propria casa. Gli abiti del cuore ci sono cuciti addosso nel passaggio delle generazioni, nelle relazioni che ognuno di noi, sin da bambino, intrattiene con la famiglia, la religione di maggioranza, gli stili di vita più diffusi, la lingua corrente, i modi con cui consumiamo e facciamo festa, celebrando i riti di passaggio del corso della nostra esistenza. Gli abiti del cuore, insomma, ci fanno abituare a stare nella società come parti integranti e integrate di essa. Le differenze e i conflitti, quando ci sono, non mettono in discussione sino in fondo le ragioni dello stare assieme. Possiamo dividerci in politica, differenziarci fra credenti e non credenti, frammentarci in corporazioni, lobbie, piccole tribù di consumatori di questo o quel prodotto di tendenza, ma tutto ciò non incide sulla rappresentazione che abbiamo della società in cui viviamo e che sentiamo come nostra.

$C^{\prime}$ è un cambiamento che, invece, va a toccare proprio il senso comune. Esso è dovuto all'arrivo di tante donne e tanti uomini, provenienti da Paesi diversi, ma da quasi tutti i continenti (Asia, Africa, America Latina, Eurasia). Venute per lavorare, queste persone hanno portato con loro le diverse lingue, culture, religioni, maniere di vivere. In pochi anni - trenta circa, meno di una generazione - siamo diventati come gli Stati Uniti: un crogiolo di culture, razze, religioni, forme di vita diverse fra loro. Non siamo gli Stati Uniti, ovviamente e non lo diventeremo forse mai.

L'idea, tuttavia, che l'Europa possa diventare sempre più differenziata culturalmente e religiosamente fa crescere la convinzione dell'insostenibilità per ragioni economico-sociali (il venir meno del sistema delle garanzie del welfare) e sempre più per ragioni culturali (e anche religiose, quando si 
tratta del rapporto con il mondo musulmano) di una società che appare loro estraniata. Sarkozy (2016) in Francia, in libro pubblicato di recente, ricorda come oggi il partito socialista di François Hollande abbia accettato un'idea che egli aveva lanciato già nel 2012: decretare la decadenza dalla cittadinanza francese per chi, di origine straniera, si sia macchiato di gravi reati contro la nazione, in particolare con atti di terrorismo. Inoltre, mettendosi di nuovo in corsa per le elezioni presidenziali del 2017, Sarkozy ha con forza ribadito che I'assimilazione sia la via maestra per stabilire un patto chiaro con gli immigrati. Assimilare è in fondo chiedere a chi porta con sé e su di sé tratti culturali diversi di non pretendere che lo Stato li riconosca in alcun modo ${ }^{8}$. Ti considero, come ha più volte notato Todorov (1984), potenzialmente simile a me, solo se la tua differenza scivola dietro il palcoscenico pubblico, divenendo un puro fatto privato e domestico. La differenza dell'altro perciò diventa un problema. Che il modello alla francese di governare società a elevata differenziazione culturale e religiosa sia ispirata in gran parte a tale filosofia politica dell'assimilazione, dovrebbe indurre a riflettere sugli effetti indesiderati e perversi che essa ha prodotto sulla società. Ma è non solo Sarkozy a pensarla in questi termini in Europa. In molti condividono il suo punto di vista, dall'Ungheria alla Polonia, dalla Danimarca all'Olanda e così via.

All'opposto dell'assimilazione c'è il differenzialismo, una forma raffinata di razzismo: ti considero talmente differente da me, che ti tengo a distanza e ti lascio riprodurre il modello di società che hai lasciato alle tue spalle emigrando e che, forse, in alcuni casi, non esiste quasi più nei tuoi Paesi di origine. In tal modo ogni migrante e suoi discendenti possono sentirsi stranieri in patria: vivono assieme in quartieri etnicamente omogenei, hanno le loro scuole, i loro luoghi di culto, si vestono, mangiano, vivono quotidianamente come se fossero in una città del Bangladesh o del Pakistan. Non m'interessa più di tanto che seguano costumi per me inconcepibili, purché si mantengano a distanza da me e dal mio mondo, dal quartiere dove vivo e dagli stili di vita che amo seguire.

In tutte e due casi la tolleranza è messa in discussione e mostra tutte le sue aporie sociali. Assimilare, infatti, implica l'idea che non possiamo tollerare che altri possano seguire costumi, pratiche religiose, modelli di famiglia e relazioni di genere che consideriamo incompatibili con i nostri standard giuridici e morali, per cui l'unico mezzo è non riconoscere in alcun modo nessun tipo di manifestazione della diversità.

Ci si può domandare, come fa il sociologo Raphaël Liogier (2016a; 2016b), se fra assimilazione e differenzialismo, che mettono entrambi in discussione un

\footnotetext{
8 Per un'accurata ricostruzione della genesi del jihadismo in Francia in rapporto alle politiche sia della sinistra sia della destra liberale, cfr. Gilles Kepel (avec Antoine Jardin). Terreur dans I'hexagone. Paris: Gallimard, 2015.
} 
tratto distintivo della storia cultura europea, non sia il relativismo la via maestra per dare nuova sostanza giuridica e politica alla tolleranza. II relativismo, infatti, non significa accettazione di tutto e di tutti senza esprimere critiche e riserve. Non è sinonimo di tolleranza integrale. Il relativismo abbassa la pretesa esclusivista e egemonica delle culture. Mette in discussione il principio secondo cui tutte le differenze sono buone perché riflesso di una specificità culturale. Nella relazione critica la ricerca di un'intesa etica è un modo intelligente, all'altezza dei tempi che viviamo, di declinare in modo rinnovato il principiotolleranza. Solo così, si può ancora cercare di governare l'inedita complessità che trova i sistemi sociali oggi in Europa relativamente impreparati a reggere le conseguenze che la diversità culturale e religiosa ha sull'equilibrio dei sistemi stessi. Le sfide maggiori riguardano in generale proprio la differenziazione sistemica che le società ultra-moderne hanno raggiunto nel corso del loro sviluppo dall'avvento della nuova organizzazione economica e sociale (il capitalismo), da un lato, e dalla forma-Stato che ha consentito l'affermazione di regimi di tipo democratico, dall'altro. La diversità culturale e religiosa può essere trattata in vari modi. $E^{\prime}$ stata trattata in Europa in base ai differenti modelli costituzionali e politici che ciascuno Stato sovrano rivendica per sé, in nome della propria specifica vicenda storica. Lungo un ideale asse di massima chiusura/massima apertura al riconoscimento delle differenze, le società europee hanno messo in pratica politiche multiple (a livello giuridico, culturale e religioso) per includere o escludere la differenza dallo spazio pubblico. Possiamo rappresentare tale varietà di modelli come se si disponessero lungo un asse massimo-minimo, assumendo convenzionalmente la via francese come il più coerente rispetto all'idea "tutti uguali come cittadini, differenti solo in privato", mentre quello inglese, a sua volta, come strategia politica che ha, al contrario, cercato di ispirarsi al principio "cittadini sì, ma le appartenenze alle diverse comunità etniche sono un affare pubblico". In tal senso, ritengo che le due formule, comunitarismo (applicato al caso britannico) e assimilazione (riferito, invece al caso francese) siano due scorciatoie socio-linguistiche che semplificano troppo la realtà: sia in Francia, sia nel Regno Unito le politiche di riconoscimento delle differenze non sono mai state così nette come a prima vista possano sembrare. Ciò che emerge, infatti, è la dimensione negoziale che le società europee hanno dovuto assumere per affrontare, senza esasperarne i contorni, i conflitti (di cui la guerra delle moschee o dei minareti è un esempio concreto) che la presenza di cittadini diversamente orientati culturalmente e religiosamente suscita. Tutto ciò alla fine mostra come la separazione fra le sfere della vita sociale, che la lezione della modernità aveva impartito alle forme politiche dello Stato moderno e alle principali organizzazioni capaci di creare solidarietà organica, entra in crisi, poiché prevale l'idea della negoziazione rispetto a quella della netta distinzione fra le sfere stesse. 


\section{Bibliografia}

ALLIEVI, Stefano. La guerra delle moschee. Venezia: Marsilio, 2010.

CAMPICHE, Roland (ed.). Croire en Suisse. Lausanne: L'Age d'Homme, 1992.

DREMAUX, Lillie. The way people look at US changed Muslim Women on life in Europe. New York Times, 01.09.2016. Disponibile su: <www.nytimes. com/2016/09/03/world/europe/burkini-ban-muslim-women.html>.

FRISINA, Annalisa. Giovani musulmani d'Italia. Roma: Carocci, 2007.

HABERMAS, Jürgen. Solidarietà fra estranei. Milano: Guerini e Associati, 1997.

HOBSBAWM, Eric. Il secolo breve. Milano: Rizzoli, 1994.

HUNTINGTON, Samuel. Who are we? The Challenges to American's National Identity. New York: Simon \& Schuster, 2004.

LIOGIER, Raphaël. La guerre de civilisation n'aura pas lieu. Paris: CNRS, $2016 a$.

LIOGIER, Raphaël. Le mythe de l'islamisation. Essai sur une obsession collective. Paris: Points, 2016b.

LUBBERS, Marcel; GIJSBERTS, Mérove; SCHEPEERS, Peer. Extreme Right-wing voting in Western Europe. European Journal of Political Research, n. 3, p. 345378, 2002.

LUCÀ TROMBETTA, Pino (ed.). II pentecostalismo, sfida alle religioni storiche. Religioni e Società, n. 72, 2012.

PACE, Enzo (a cura di). Le religioni nell'Italia che cambia. Roma: Carocci, 2013.

PACE, Enzo. Perché le religioni scendono in guerra? Roma-Bari: Laterza, 2004.

PACE, Enzo; BUTTICCI, Annalisa. Le religioni pentecostali. Roma: Carocci, 2010.

Papa FRANCESCO. Discorso al Parlamento Europeo. Strasburgo, 25.11.2014. Disponibile su: <https://w2.vatican.va/content/francesco/it/speeches/2014/ november/documents/papa-francesco_20141125_strasburgo-parlamentoeuropeo.html>. Visitato il 15.07.2016.

PIRJEVEC, Joze. Le guerre jugoslave. Torino: Einaudi, 2001.

SARKOZY, Nicolas. La France pour la vie. Paris: Plon, 2016.

STOLZ, Jörg (ed.). (Un)Believing in Modern Society. London/New York: Routledge, 2016.

TODOROV, Tzvetan. La conquista dell'America. Torino: Einaudi, 1984.

Articolo ricevuto il 04.04.2018

Accettato per la pubblicazione il 27.06.2018

Received for publication in April 04 ${ }^{\text {th }}, 2018$

Accepted for publication in June $27^{\text {th }}, 2018$

ISSN impresso 1980-8585

ISSN eletrônico 2237-9843

http://dx.doi.org/10.1590/1980-85852503880005302 